Prilin and Incann, thene is no longer roam for doubt on the mabject. Thking the mean of all Dr. Dary's experiincents, the specific grovity of venous blood is ascertained to bo 1.053, that of artarial blood but 1.050. The specific grevity of the carum of venous blood, again, he found in the mean to be 1.026, that of the serum of arterial blood 1.028. In reperting these inquiries into the specific grevity of the different kinds of blood, I very constantly found the serum of renous blood of somewhat higher density than that of arterial blood; and even when, through imperfection of apparatus, the difference between the two seemed very slight, or was even inappreciable, I still found, on putting the two kinds of serum into an apparatus for showing endosmose, that a current of considerable force was established from the arterial to the venous side. And this is the process, in fact, which, as carried on between the fluids that transude the arteries, for the purpose of nutrition and vital endowment, and the contents of the reins in the living body, constitutes renous absorption; the condition indispensable to this, viz., the higher density of the blood in the veins or returning channels than in the arteries or efferent vessels in all the peripheral parts of the body, being mainly due to the influence of the sudoriparous glands distributed through the texture of the common integument. This view of the function of the skin will be seen to harmonise perfectly with the fact that the grand product of the action of the skin is simple water. It also explains satisfactorily the fatal effects of the entire suppression of the cutaneous transpiration, as in the experiments of Fourcault and of Becquerel and Breschet. Finally, it sheds a new light on the cause of the great amount of disturbance in the general health that follows every even partial interference with this truly vital function.

It would have been easy for me to have greatly extended this communication, and to have shown the views it involves in a variety of practical applications; but I have purposely aroided doing more than exposing an essential idea which has now for many years possessed my mind, and directing the attention of the intelligent members of the Medical Society of London to a subject which I feel assured is pregnant with much that is of the highest importance in the theory and practice of medicine.

Barnes, May 1854.

\section{ON THE TREATMENT OF STRANGULATED HERNIA AFTER OPERATION.}

By EDWIN MORRIS, M.D., F.R.C.S., Surgeon to the Spalding Union Infirmary.

[Read at the Annual Meeting of the Midland Branch of the Provincial Medical and Surgical Association at

Lincoln, June 1, 1851.]

Arthodar the provincial surgeon is not often, I am happy to say, called upon to perform the operation for the relief of strangulated hernia, it behoves us all to be well acquainted with the nocessary measures and caution to be taken for the safe reduction of the incarcerated bowel when the painful necessity occurs, and to be thoroughly conversant with the most approved and judicious plan of treatment after the operation itself has been successfully doue. It is not my intention to detail the operative procedure, or to enumerate the anatomical difficulties that may arise; inasmuch as such a proceeding would be presumptuous in me, and a waste of valuable time. I proposetherefore to take the management of a ease of hernia immediately after operation. It will be necesary therefore to give briefly the treatment which others hare formerly recommended, before explaining that of some modern surgeons and my own. The circumstances that I have deroted a great deal of my time to the consideration of this all-important subject, and also that I have had some experience, more than usually falls to the lot of the country eurgeon, must be my apology for introducing this interesting point to your notice.

Upon referring to the writinge of the lato Bir A. Cooper, then whom \& greater authority on this subject does not exist, I find that he says in his Lectures on the Principlin and Practice of Surgery, "In five or six hours after the operation, give a little sulphate of magnesi or castor-oil. When a patient has two or three motions, the surgeon thinks be will do well; but the danger is not orer, and it will be neceseary to keep up a free discharge from the bowels by opening medicines, or the patient will die. The history of a case is generally this : in four or five hours after tho operation, the patient has a motion, and in the course of twenty-four hours, two or three, and he will be supposed to be doing well. On the following day there will be no motion. the abdomen is tense and tender to the touch, and vomiting comes on. Were I to tell you how many I have known do well for three days, and then die after all, I should enumerate a long list. Great danger is to be apprehended for some time afterwards, and even those who have two or three motions within twenty-four hours often die." Such was the opinion and practice of the most eminent man of his day, viz., to give a purgative five or six hours after the operation; a practice which was universally adopted, and which doubtless, in a great measure, caused the long list of deaths which Sir Astley was capable of enumerating.

Mr. Lawrence, too, of St. Bartholomew's, who stands second to none in his knowledge of this particular branch of surgical pathology, states in his Treatise on Ruptures, a work of grest research, affording a mass of valuable practical information: "When the operation is finished the patient should be placed in bed, and allowed to remain there quietly for some time ; a little tea, thin gruel, or other diluent being given if it is desired. The pain is lessened and the vomiting ceases after the operation; sometimes the bowels are spontaneously relieved, and a considerable abatement of the symptoms in general ensues. More commonly it is necessary to solicit the action of the intestinal canal by aperients and injections. If, therefore, the bowels should not have been relieved in three or four hours, a few grains of calomel may be given in a pill; or two pills may be administered, consisting of calomel and the compound extract of colocynth in equal parts. The sulphate of magnesia may be given afterwards, in the dose of two drachms or of one drachm, in the infusion of roses, or in a mixture of mint-water and common water, and this should be repeated every three or four hours until the bowels are freely relieved. We must repeat those or similar means, and persist in their employment until the canal is completely unloaded." He also adds in a note at the bottom of the same page, that "M. Velpeau recommends the exhibition of purgatives after the operation, and dissents expressly from the opinion of Dupuytren and others, that these remedies increase the probability of inflammation." The work from which I have just quoted was published in the the year 1838. Whether Mr. Lawrence still holds the same opinion, or pursues the same practice, I am unable to say.

I wish to confine my remarks more especially to the consideration of purgatives, and to endeavour to clear up the disputed point whether it is better to administer them immediately after the operation for sirangulated hernia, or to wait and leave nature to take her own course. My attention was first directed to this important point by the following interesting case.

CA8E. A gentleman, a valued friend of mine, aged 76 , went to Brighton for change of air and sea-bathing. During the time he was there, he was attacked with the symptoms of strangulated herria. The late Mr. John Lawrence was sent for (a man distinguished for his ability as a surgeon, and for whose untimely fate many will mourn); and, after having tried the various means usually had recourse to, he was unable to reduce the rupture. The operation was proposed, and submitted to without a murmur. The constricted bowel was relieved, and the more urgent symptoms immodiately subsided. No purgative, and, indeed, no medieine of any hind was given, and it was seven days after the operation before the bowels were relieved, when an evacuation took place spontaneously, and without the least inconvenience or pain. No inflammatory action took place, and the 


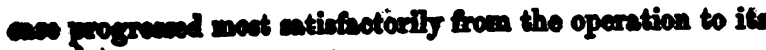

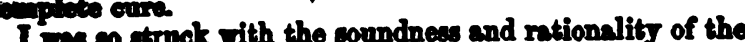
Inotion, that I made further inquiries, and found that this Fin the practice Mr. J. Inwrence nniversilly adopted, and with growt success. He had the history of no fower than tweenty-fire caees in which this plen was pursued, with the same satisfactory results. He also stated that it was his intention to publish, for the benefit of the profession, the whole of the above cases in detail. But, alas! how uncertain is life! within a fer weeks of this time Mr. Iawsence, in the midst of a brilliant and honourable career as surgeon, was himself the subject of obstruction in the bowels; and, after several days of severe suffering, was suddenly required to pey the great debt of nature. Thus the profession sustained a great loss, and society was deprived of the services of an observant and practical surgeon.

The non-administration of purgatives after the operation for strangulated hernis is evidently gaining ground; and, indeed, has been especially insisted upon by more recent surgical writers. I find that Mr. Skey, in his Operative Surgery, says, "As regards after treatment, it should be, for the most part, passive. If the sickness subside, and the bowels act quickly, it is a farourable omen of recovery; but should some hours elapse without action, it is no excuse for the administration of drastic purges. It is difficult to lay down any specific rule on this subject, so various may be the indications; but I believe it is better to leave a patient alone. If, however, at the expiration of twelve or eightoen hours no action appears probable, a mild aperient may be given, in the form of manna, with some compound decoction of aloes, or mild form of neutral salts with some tincture. The intestine may also be excited to action by enemata of warm water with salt, or other mild purgatives, not to be repeated with too great frequency." Such is the opinion of Mr. Skey, who is evidently in farour of the nonpurgative plan. Although he does not entirely negative the use of purgatives, he admits that "it is better to leave a patient alone". I am inclined to have great respect for any plan of treatment recommended by Mr. Skej, placed, as he is, as surgeon to one of the largest hospitals in London. His opportunities of witnessing and conducting the treatment of surgical cases are immense, and such as rarely fall to the lot of others.

In the seventh volume of the Medical Timesand Gazette, for 1853, is published the history of T200 Cases of Inguinal Hernia, in which the sac was pushed back with the intestine, by Mr. Paget. They are given in detail for the purpose of shewing the result of this procedure, and not with regard to their after treatment. But, as Mr. Paget has treated them after the manner I am adrocating, I shall take the liberty of quoting so much as may be necessary towards elucidating and forwarding my views embodied in this paper.

The first case was that of a strong healthy-looking waterman, aged 23 years. The patient spent the greater part of the twenty-four hours following the operation in sound sleep; he had no romiting or action of the bowels; no mediclne was giren, and he took nothing but little bread, tea, and water. On the second day, the anme abstinence from food was all day voluntarily observed, and no medicine was given. On the fourth day his bowels were still unmored; a common enems was injected, and coon returned with a free evacuation of hard, and some fluid freces: he took bread and milk during the day, and felt and looked nearly well. From this time his recovery was uninterrupted.

The second case was that of a man 41 years old, a tall, nuscular cabinet-maker, of pallid, unhealthy appearance, and of rather intempernte habits. In the twenty-four hours following the operation the patient often slept for short tien. The abdomen was orery where coft, pliant, and painIne upen precure, except about the plece of opecation. No

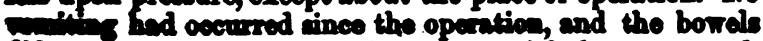

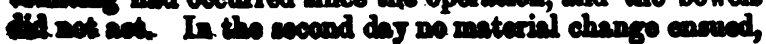

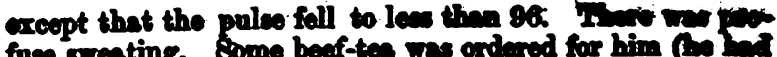

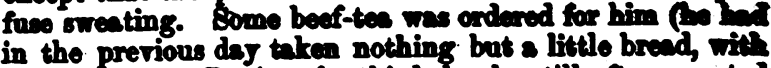
in the previous daj taken nothing but s lithe broad, wented profusely. He looked and folt more feoble and doperened. In the evening, four ounces of wine with more beef-ten tres ordered. On the fourth day, he said that ho had felt at once better for the wine, and slept soundly, and with rofreshment after it; the bowels remained inactive, and common enems was directed; after the enems the bowels acted freely twice, and from this time his progress to complete recovery was not interrupted.

So much for these two interesting cases, 80 grophicalls and admirably described by Mr. Paget. Nothing could show more forcibly and satisfactorily the excellent plan of leaving nature to herself after the operation, than the abore cases. No anxiety was evinced or expressed by Mr. Paget in consequence of the bowels not having acted, nor did ho deem it necessary until the fourth day to have recourse to any means for the purpose of exciting the borels to action; and, indeed, not even then (and with becoming respect to Mr. Paget do I state it) do I think it was needful, for in both instances, had time been given, the bowels would doubtless have acted spontaneously.

In connexion with this subject, I must refer to the annual oration which was delivered last March before the Medical Society of London, on The Improvements of Modern Surgery, by Mr. Henry Smith. Speaking of hernis, in p. 16, he states: "The other point alluded to is, the treatment of cases after the operation has been performed, and here a great improvement has taken place within the last fer years, in consequence of a more correct lnowledge of the pathology, and of the treatment of abdominal inflammations in general; the majority of practitioners now recognise the great importance of keeping the inflamed parts at perfect rest, and of tranquillising the nerrous and vascular system. It is no longer considered necessary that a portion of bowel, which had been constricted and irritated for hours or days, should, immediately after it has been liberated, be further irritated and exposed to mischief by the action of purgatives, nor is it deemed prudent that large abstractions of blood should be made for the purpose of preventing or remedying peritoneal or intestinal inflammation. Thus, instead of giving castor-oil or colocynth soon after the operation for hernia, the surgeon allows nature to take its course, giving time for the injured intestine to repair itself; and if signs of inflammation do come on, he depends upon opium as his shoet anchor, instead of prostrating his patient by copious, local, and general blood-letting, or by profuse salivation." The above pertinent and laconic remarks of $\mathbf{m y}$ friend, $\mathbf{M I r}$. Smith, relative to the administration of purgatives after the operation for hernia, are 80 much in accordance with my views, and are expressed by ono so deservedly high in his profession, as to make it almost superfluous for mo to proceed further. But I trust I shall be pardoned, and not considered egotistical, if I detail the result of $\mathrm{my}$ own practice in the treatment of hernis after operation.

It was not long after I had made up my mind as to the course I should pursue, before an opportunity presented itself.

Casi I. A married female, aged 38, was three weeks after her confinement attacked with the symptoms of strangulated hernia. A small recent femoral hemis was discovered on the right side: the usual means were adopted for its reduction, but without effect. The operation was performed, and the constricted bowel was returned without much difficulty. The integuments were evenly adjusted, and kept in appoaition by means of sutures and adhesive plaster. The horizontal posture was strictly enjoined, und no medicine given. The next day I found that the romiting and hiccough had quite subsided; there was little or no pain; and the bovals had not acted; indeed, there was not a symptom present to canse me to feel at all anrious for the bovel to act. I docided not to give a purgative, but to leave nature to twito her natural courne. All the divtrening and alarming bis

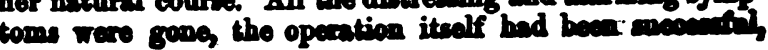


and the intestines were again in their natural state. The ace progreseed most frourably up to the evening of the thind day, when, for the first time after the operation, the bowels were, without the aid of purgatives, naturally and. atisfactorily relieved. From this time her recovery was rapid; and by the end of the month she was quite well.

CAsE II. The next case was that of a labouring man, aged 56 years. He had not been the subject of rupture previously, and was ignorant of the cause of his vomiting and pain. Upon examination, I found that he had a small recent hernis on the right side; attempts were made to reduce it, but without avail. I proceeded to operate, and readily reduced the bowel in the usual way; the parts were brought together by sutures and adhesive plaisters, and dossils of lint and bandage were applied for the purpose of producing slight pressure. No medicine was given. He passed a comfortable night after the operation; the vomiting had ceased, as well as the pain and hiccough; he had taken tea and toast. On the second day there was tenderness about the wound, of which he complained. A dozen leeches were applied, which considerably relieved him. The bowels were not yet relieved. He gradually improved up to the fourth day, when in the evening the bowels were copiously relieved. His progress to recovery afterwards was most rapid and satisfactory.

CA8E III was one of femoral hernia, in an old lady aged 70 years. No purgative was given, and the bowels acted on the second day. On the fourth day she suddenly began to sink, and expired after a few hours, without any assignable cause.

CAsE IV. A similar case to the above occurred last Sept. in the practice of Mr. Agar, of Crowland, in this county. The patient, a man, was upwards of 70 years, and was the subject of strangulated inguinal hernia. He suffered a long time before he would submit to the operation; but I at last prevailed upon him to do so. I had little difficulty in fiberating and returning the incarcerated gut. $\mathrm{He}$ was treated after the same manner as the above cases. The next morning the bowels were relieved plentifully. The patient residing a considerable distance from me, I did not see him again after the operation. Mr. Agar, however, kindly gave me every particular. It appears that all went on well until the second day, when a collapse suddenly came on, and after a few hours he expired.

I much regret that in neither of the above cases was a post-mortem examination permitted. This sudden sinking and expiring after the operation, I believe, is not an unfrequent occurrence, and without any apparent cause, in very old people. I am inclined to attribute it to the failure in the vis vitce of aged persons, which is unable to contend with and resist the great havoc and shock the system receives during the time of the strangulation, from the distressing and agonising symptoms necessarily attendant on 80 formidable a matter as constriction of the intestines, and from the operation itself. I never heard or read of a similar case happening in young persons.

I shall only detail one other case, before making some general and concluding remarks. I could give several more cases, showing clearly and indisputably the excellence of the practice of avoiding purgatives after the operation for strangulated hernia, and pursuing a passive plan of treatment.

CAsE v. The case I allude to is that of a lady, aged 51 years, who had been the subject of irreducible femoral hernia for years, and had at times suffered great pain and inconvenience from it, having had several attacks of sickness and constipation, but was always, after having had an evacuation from the bowels, relieved. From motives of delicacy, she had not consulted her medical attendant. Last summer she was seized with one of her attacks of sickness and pain in the abdomen; her stomach rejected everything. Finding that her usual remedies did not afford relief as before, she sent for her surgeon, who soon informed her that she was suffering from strangulated femoral hernis. I was requested to see her; and as all known means had been had recourse to without any benefit, I immediately operated, and readily returned the bowel. The sac I was unable to return, in consequence of its having formed strong adhesion to the surrounding parts ; and as it interfered with the proper and accurate adaptation of the integuments, I removed a piece with the scalpel. The wound was dressed in the usual way, and no purgative was given. In consequence of the great distance from me I did not see this patient again, but I was favoured with a most satisfactory report. The bowels acted on the second day spontaneously, and not a bad symptom followed the operation.

The object of this paper is especially to prove the desirableness of the plan of not giving purgatives after the operation for strangulated hernia. By their action, great and unnecessary irritation is produced within the bowels, at a time, too, when rest and quietude are most needed and beneficial; for we must not, in our anxiety, overlook the important fact, that the intestine has suffered already sufficient irritation and injury from the violent and continued constriction to which it has been subject prior to the operation. Surely then it is better that it should remain at rest, so as to relieve itself of an unusual state of congestion, and thus be afforded the chance of assuming its normal state again. In the cases I have related, the bowels generally acted about the third or fourth day.

We will now trace the treatment of a case of strangulated hernia to its cure. The operation having been successfully performed, and the constricted bowel having been safely returned within the cavity of the abdomen, the edges of the wound must be brought neatly together by means of sutures, taking care to include nothing but the skin, which must be even and in exact apposition. Our object is to produce adhesion, and the more we can effect of this, the greater will be the advantage gained after this has been done; it is better to exercise slight pressure upon the part by means of dossils of lint, and a bandage. The patient must be strictly enjoined to keep the horizontal position; and when the bowels are relieved, he nust use a bed-pan. A case is related by Mr. Cline where this was not attended to. He directed that his patient should not quit his bed; a little time after the operation, however, he did just get out of bed, and whilst upon the night-chair the bowels forced their way into the sac, and displaced the dressings. The above caution therefore is not superfluous, but highly necessary. The sickness having now subsided, a little gruel may be allowed. On the second day after the operation, the sutures may be removed; for, if adhesion have not taken place within this time, most assuredly it will not. The wound may now be dressed with a little simple cerate. Should it, however, inflame or suppurate, a few folds of lint dipped out of tepid water may be applied, and occasionally changed. Over this must be placed a piece of oiled silk. If there is much ferer present, a simple saline may be given every three or four hours. The bowels may not yet have acted; a purgative, however, must not be administered, lest the intestines should not have recovered their tone. Should sickness continue or supervene, effervescing medicine must be given.

The plan of treatment I have described must be continued until the wound has completely healed, never omitting to apply slight pressure over the dressing by means of a pad of lint and bandage. After the wound is healed, the parts will remain for some time very tender, so much so that the patient cannot bear the pressure of a truss. Now it is essentially necessary that a truss should be worn afterwards; and until the parts are in such a condition as to bear the pressure of one, the use of a pad and bandage must under no circumstances be neglected; for, although the operation has relieved the strangulated gut, and enabled it to be returned within the cavity of the abdomen, still the liability to a return of the hernial protrusion remains, to guard against which a truss must be always afterwards worn.

The bowels will generally act spontaneously on or before the fourth das. Should they not, however, there need be no apprehension lest mischief should ensue ; for, in the absence of any unfarourable symptoms, the fact is simply unimportant. Four dajs having been allored to elapese since 
the exantion, the bowels will now hwe reoweed their nomal condition, and the great risk of infiamantory setion will have passed away. On the fifth day, therefore, should no erreustion have taken place, a simple enems of warm water my be thrown up the rectum; and this is almost certain to be followed by a satisfactory relief. Should it not, howerer, the injection may be repeated the next day. $\Delta$ fter the bowels have been relieved, a nutritions diet may be allowed. The patient usually recovers within a month of the operation.

Such is the history and treatment of a farourable case of strangulated hernia after operation, but matters will not, in spite of every precaution, progress so smoothly as I have depicted on every occasion. Sometimes, a few hours after the operation, symptoms of an alarming character will come on; and it will require our best energies and attention to combat and subdue them. The most formidable occurrence which we may dread, and may be called upon to treat after the operation, is peritoneal intlammation. It behoves us, therefore, to be on our guard, so as to at once attack the first appearance of this alarming and often fatal sequent of the operation. The symptoms of peritonitis are readily recognised. There is grcat uneasiness orer the whole of the abdomen, with pain and tenderness about the wounds; the pulse is small, hard, and frequent; the skin is hot and dry; there is great thirst; the tongue is dry and brown down its middle; the knees are drawn up; the belly is swollen, and very painful upon pressure; the breathing is short, and sometimes sickness happens. Such are the leading symptoms when peritoneal inflammation is present; and nothing short of the most vigorous antiphlogistic treatment will arrest this fatal affection. We must remember that it is traumatic peritonitis we have to deal with; and, as far as my experience goes, I believe it to be much more acute and more fatal in its termination than idiopathic peritoneal inflammation. The patient must be bled largely and freely from the arm, and by the application of a dozen or even two of leeches to the abdomen; and a full dose of opium must be given. Should the symptoms not yield to the above treatment, mercury must be administered, and the system brought under its influence as speedily as possible. I wish especially to draw attention to the fact, that immediately after bleeding I have recommended a dose of opium, giving it the priority of mercury. I admit that this is contrary to the practice of the late Sir A. Cooper, Mr. S. Cooper, Liston, Lawrence, and some modern surgical writers; but to use the words of Mr. Skey, in his recent work on Operative Surgery, "I look forward to the time when its employment in inflammation will raise less alarm in the public mind than it does at present"! Then, again, Mr. Henry Smith, in his pamphlet on The Improvements in Modern Surgery, speaking of the treatment of hernia after operation, says that " if signs of inflammation do come on, he depends upon opium as his sheet anchor, instead of prostrating his patient by copious, local, and general bloodletting, or by profuse salivation." This is strong and unmistakeable language respecting the value of opium, and spoken by one whose position and experience entitle his opinion to the greatest respect. The researches of modern pathologiste indisputably show us that inflammation is not simply a greater determination of blood to a given part, or an unnatural action of the vessels themselves, accompanied by an excited state of the whole vascular system, but that the blood itself is in a morbid state, and is the immediate cause of many inflammatory affections; and that the plan of depressing and lowering the system by general and local bleeding, and giving powerful medicines, is wrong and may be fatal. I dare say many have observed the fact sometimes, when treating a case of acute inflammation, that the acute lancinating pain has not always yielded to the abstraction of blood, but has at once been subdued by a full dose of opium.

In peritonitis, warm applications to the abdomen will be found both serviceable and agreenble; and this is beat done by fannels wrung out of bot water. A simple saline mixture may be giren, and warm water clyaters thrown up the rectum an often as may be necencary to procure an eracus-

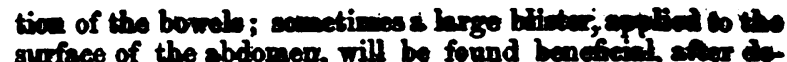
pletion has been carried as far as is thought predent. I must confees, however, that I here not mueh fnith in their efficacy. Peritoneal inflammation will generally be found to yield to the plan of treatment I have just detailed, if pursued in a determined and vigorous manner.

Since writing the above, my attention has been directed to the history of a "case of herniotomy" published in the Associa tion Jouras of May 12th, by Dr. John MeIntyre, which so clearly shows the direful effects that may follow the administration of purgatives after the operation for hernia, that no apology, I am sure, is needed from me for introducing it here; and I must add that the author, with a candour that does him much credit, and which all scientific investigators will applaud, at once admits his error, and promises to avoid the purgative plan of treatment for the future. I shall only quote such portions of this unique case as may be necessary for my purpose.

James Cooper, aged 67, was the subject of strangulated femoral hernia; the usual means were tried to reduce it, but without effect; on December $20 t h$, it was deemed unsafe to try the taxis further, the operation was proposed, to which he willingly acceded. The operation was done in the usual way, and the intestine replaced within the cavity of the abdomen; very little blood was lost during the operation, and no vessel required ligature; he immediately felt relieved. The wound was united by two or three points of suture, a compress and bandage applied, and he was placed in bed. The pulse was 70 .

December 21st. He had passed a good night, and felt comfortable; there had been no pain or vomiting; he had passed urine twice; the bowels had not acted. He was ordered to have a large enema of gruel, and two scruples of Epsom salts every four hours. 8 P.x. The bowels acted freely three times from the enema; he had vomited twice, and now felt sick. You will observe now that the patient's troubles begin; purgatives are given within twelve hours after the operation. He complains of uneasiness round the upper part of the abdomen, which is distended from flatulence. There was no pain at the wound, the skin hot, great thirst, pulse 72 . He was now ordered to take a calomel pill at once, and half an ounce of castor oil.

Dec. 22. The oil was rejected. "I was called to him," says Dr. McIntyre, "at four o'clock this morning, on account of vomiting and severe pain at the scrobiculus cordis, the result, I believe, of another dose of aperient mixture that had been given by my assistant to him. At 11 P.x. pulse 78, tongue moist and somewhat furred; a calomel and opium pill was ordered every four hours.

"Dec. 23. He had passed a good night; the bowels felt as if they would be relieved; was free from pain. I undid the bandage; the wound looked well. The pills were omitted at 6 P.M. The bowels had acted four times; the last motion was 'bloody and slimy'; the abdomen very collapsed; otherwise he remained in the same state. Thirty drops of laudanum in a mucilaginous mixture was given every four hours.

"Dec. 24. The night had been disturbed by repeated actions of the bowels, dysenteric in character, and accompenied with tenesmus. An enema, containing half a dram of laudanum, was ordered, and the anodyne mixture continued; in the evening the bowels were quieter; the opium in mixture was omitted.

"Dec. 26th. He felt better. The bowels had acted twice since last evening ; four times since last report. The wound was rather inflamed; I removed the sutures, and applied water dressing; the pulse was 76. One ounce of chalk mixture, with five drops of tinct. opii, was given every six hours. On the 29 th he had paseed a rery restless night, the bowels having been moved nine times; the stools were dysenteric, and apparently mixed with pus; an astringent enema, containing half a dram of laudanum, was giren, and the chall mixture continued in the evening. The bowel had acted three times; the stools were feculent, and mixed 
with blood and mucus. He had no pain, and felt comfortable; the enema was repeated. 318t. The bowels had not been mored; he felt more comfortable in every respect. The reports from this date tell of his gradual recovery."

The author then states in his remarks : "The muco-enteritis, I believe, arose from a combination of causes : from the use of the aperients; from the specific action which mercury has upon the bowels of some individuals on its introduction, however slightly, into the system. The markedly beneficial influence of the opium throughout the case especially deserves notice, adding as it does to the evidence in favour of its use in strangulated hernia, and in abdominal injuries and diseases generally. Most recent writers in the after-treatment of cases of herniotomy advocate the propriety of conceding rest to the bowel to enable it to recover the injury which its structure or function may have sustained, and this, not only by abstaining from the use of aperients, but by administering opium to accomplish it. The duration of this repose will vary of course with individual cases, and will depend upon whether the patient has been brought under its influence during the trial of the taxis. In the present case, the patient continued drowsy after the operation was completed, and none was given until the biswels were relieved. Mr. Guthrie recommends that one grain should be given two or three times a day, according to its effect, for the first two days, and be followed up by a large emollient enema at the expiration of that time. I coniess that I was not sufficiently delivered from the trammels with which the long inculcated orthodoxy of the aperient treatment had entwined me, to wait so long, but when the vomiting and pain that occurred on their administration supervened on a hitherto favourable condition, I repented of my adherence to past teaching, and resolved in future cases to adopt that plan unhesitatingly, which modern science has shown to be the most rational, and modern experience the nost successful treatment."

Did time admit, I could say much more in farour of the passive plan of treatment after the operation for hernia. I am convinced that the administration of purgatives within the first twelve hours after the operation is not only unnecessary, but absolutely dangerous; and I have not the least doubt there are gentlemen present who have witnessed the beneficial effects of the former mode of treatment, and the deplorable results of the latter. In conclusion, permit me to observe that I am well aware I must yield to many present in point of opportunity and ability to treat this practical subject in the manner that its vast importance demands; but I will yield to no man in the sincerity of $m y$ intentions. The few imperfect remarks that $I$ have made are intended for comparison with the experience of others in the great field of practical surgery.

The life of one man is too short for him to do much towards advancing his profession as a science, but much may be done by comparing notes, for by this means the healing art may be placed upon a surer basis ; and should my humble efforts in the least degree assist in promoting this much desired result, my labour will not have been in vain, or your time needlessly wasted.

Spalding, June 1854.

\section{INQUIRY INTO THE PROPRIETY OF OPENING THE BOWELS SOON AFTER THE OPERATION FOR STRANGULATED HERNIA.}

\section{By JOSEPH SAMPSON GAMGEE, Esq.}

Thodar, from the days of Franco, the most renowned surgeons have specially studied all matters relating to herniaso much so, indeed, that the reputation of sereral of the most illustrious among them is mainly based upon those investigations, and that more may be said to be known of this disease than of any other in the whole range of surgerythere are yet some important points upon which opinion is divided.

Having already submitted to the profession, in the pages of this periodical, a few considerations upon therdativemeciti of the intra-and extra-peritoneal methods of herniotomy, a rexed question of great practical moment, - I shall endear vour to contribute to the solution of another no less disputed and momentous question: Is it advisable, where they do not act spontaneously, to open the bowels soon after the operation for hernia; or is it, on the contrary, desirable to favour the quietude of the alimentary canal?

This question has often perplexed me in watching cases of hernia: and the perplexity has been only augmented in my endeavours to remove it, by consulting the opinions of the most renowned surgical writers. One line of practice is insisted on by Louis, Samuel and Astley Cooper, Hey, Lawrence, Richter, Velpeau, and Syme; a totally opposite one by Dupuytren, Liston, Miller, Hancock, and others. Velpeau strenuously recommends purgatives after the operation for their power in preventing inflammation: their undoubted tendency to excite and aggravate it is the reason which Dupuytren alleges for objecting to them. The propriety of early administering them is regarded by Mr. Lawrence as one of the most unequirocal results of experience and the plainest dictates of common scnse: Mr. Hancock insists that the most unequirocal results of experience, and the plainest dictates of common sense, no less than doctrine, prove the injurious effects of purgative medicines after the operation for strangulated hernia.

Considering the great experience of the men who have defended each side of the question, and the certainty that the very opposite practices which they enjoin must in particular cases be productive of mischief, it becomes interesting to inquire in to their respective claims to assent, by an examination of the reasons and facts they adduce in their support.

Professor Miller teaches, that "after successful reduction by operation, the same treatment is required as in the case of simple taxis.... bland enemata, but no purge by the mouth, however simple, until many hours have elapsed; otherwise dilatation with obstruction will take place above the palsied portion of intestine, and the patient will probably sink under the symptoms of ileus."* Since dilatation with obstruction of the palsied portion of intestine is, to a greater or less extent, the condition par excellence of every patient whose bowels have not acted after his being the subject of strangulated hernia, there is no need of purgatives to induce it; the only question can be whether they would aggravate it: theoretically, we do not see how they can do so ; and, practically, we know that they almost always succeed in speedily removing the obstruction,-reduction, I presume, of course, to have been effected prior to their administration. If in such cases purgatives exercise an injurious influence, it must be of altogether an opposite character to that attributed to them by Professor Miller; by so much stimulating the intestinal functions as to excite or aggravate already existing inflammation, or by determining the rupture of an ill-conditioned piece of bowel. So far from sharing the fear of the Edinburgh professor, Vidal, after remarking that many surgeons, influenced by theoretical preoccupations, have too exclusively condemned the use of purgatives after the operation ? $r$ hernia, states that he has with great benefit had recourse to the exhibition of croton-oil to overcome the excessive sluggishness of the bowels in some old subjects. It must be observed, however, that Vidal expresses himself dogmatically, and adduces no reasons or sufficient facts in defence of his own, and in condemnation of the opposite practice. $\dagger$

For precisely the same reasons as Mr. Miller interdicts purgatives, Richter and Louis recommend them. "If the strangulation," to use Richter's words, "depend upon an impaction of fæces, the intestines are so weakened after the operation as not to be able to clear themselves of their contents, which are a source of irritation to them, and interfere with the patient's well doing. Small doses of Epsom salts and clysters speedily produce an evacuation, and got rid of the unpleasant symptoms." +

- Practical Surgery, 2nd edit., p. 364.

+ Traité de la Pathologie Ext. et de Méd. Opératoire, rol iv, p. 189.

† Trattate delle Ernle. Prims Traduzzione Italiana, Milano, I002, p. 188. 